\title{
THE LINEAR DIFFERENCE-DIFFERENTIAL EQUATION WITH LINEAR COEFFICIENTS
}

\author{
BY \\ BARBARA G. YATES
}

1. Introduction. The linear difference-differential equation whose coefficients are linear functions of the independent variable is of the form

$$
\sum_{\mu=0}^{m} \sum_{\nu=0}^{n}\left(a_{\mu \nu} x+a_{\mu \nu}^{\prime}\right) y^{(\nu)}\left(x+b_{\mu}\right)=v(x) .
$$

We suppose $x$ to be a real variable,

$$
m \geqq 1, \quad n \geqq 1, \quad y^{(0)}(x) \equiv y(x), \quad 0=b_{0}<b_{1}<\cdots<b_{m},
$$

$v(x)$ a known function, and $a_{\mu \nu}, a_{\mu \nu}^{\prime}$ any numbers (real or complex) such that $a_{m n} \neq 0$ and $a_{m n}^{\prime} / a_{m n}$ is either nonreal or positive. This equation is a particular case of the general linear equation (see Wright [7]), where the coefficient $a_{\mu \nu} x+a_{\mu \nu}^{\prime}$ in (1.1) is replaced by a known function $A_{\mu \nu}(x)$, and also of the linear equation with asymptotically constant coefficients (see Wright [8], Cooke [1]), where $A_{\mu \nu}(x)$ tends to a finite limit as $x \rightarrow \infty$. Reference was made to (1.1) in Wright and Yates [10], which deals with a generalization of the Bessel function integral involved in Lemma 4 of this paper, and the equation was previously considered by Hoheisel [3], who was chiefly concerned with the existence and uniqueness of its solutions. Mirolyubov [4] has also discussed (1.1), taking $x$ to be complex, but merely stating in general terms (without proof) that a solution may be expressed as the sum of two contour integrals $\left({ }^{1}\right)$.

We consider here the asymptotic expansion for large positive $x$ of the solution of (1.1) satisfying certain boundary conditions. In all our results only the leading term of the expansion is given, but further terms are of course calculable. As a preliminary to more general considerations, Cooke [1, Theorem 1.1] has established, for the values $m=1=n$, a result similar to Case (a) of Theorem 1 of the present paper. His method of proof is essentially that used here, although the details are worked out somewhat differently. However, the conditions he imposed exclude the possibility of an asymptotic expansion of the form of Cases (b) and (c) of Theorem 1( $\left.{ }^{2}\right)$.

I should like to express my gratitude to Professor E. M. Wright for sug-

Received by the editors December 15, 1954.

(1) Added in proof. Details and proofs were published later (A. A. Mirolyubov, Math. Sbornik vol. 34 (1954) pp. 357-384 (Russian)).

(2) Certain calculations of elementary character, given only in outline here, will be found in full in a thesis presented for the degree of Ph.D. of the University of Aberdeen in 1952. 
gesting the problem, and for his guidance and advice throughout the work.

2. Notation and statement of theorems. As boundary conditions we suppose given the values of $y^{(\nu)}(0)$ for $\nu=0,1, \cdots, n-1$ and of $y^{(n)}(x)$ in the initial interval $0 \leqq x<b_{m}$, and we assume that $y^{(n)}(x)$ is $L^{2}\left(0, b_{m}\right)$ and $v(x)$ is $L^{2}\left(0, x_{0}\right)$ for all finite $x_{0}>0$. We define $y^{(\nu)}(x)(x>0, \nu=0,1, \cdots, n-1)$ by

$$
y^{(\nu)}(x)=y^{(v)}(0)+\int_{0}^{x} y^{(v+1)}(u) d u,
$$

so that by the conditions on $a_{m n}, a_{m n}^{\prime}$ and Theorem 2 of [7] it follows that $y^{(n)}(x)$ is $L^{2}\left(0, x_{0}+b_{m}\right)$. Hence $y^{(\nu)}(x)$ is $L\left(0, x_{0}+b_{m}\right)$ for all $\nu$.

Using the notation of [8], the set $\mathcal{X}$ is defined to be the set of the real parts of the zeros of the function

$$
\tau(s)=\sum_{\mu=0}^{m} \sum_{\nu=0}^{n} a_{\mu \nu} s^{v} e^{b_{\mu} s}
$$

together with their limit points. For any function $f(x)$ which is $L^{2}\left(0, x_{0}\right)$ for all finite $x_{0}>0$, the function $\omega(f)$ is defined by the condition that $f(x) e^{-\sigma x}$ is $L^{2}(0, \infty)$ for all $\sigma>\omega(f)$ but for no $\sigma<\omega(f)$, with $\omega(f)=-\infty$ if $f(x) e^{-\sigma x}$ is $L^{2}(0, \infty)$ for all $\sigma$ and $\omega(f)=+\infty$ if there is no $\sigma$ for which this is true, while

$$
\omega_{n}(y)=\max _{0 \leqq_{\nu} \leqq_{n}} \omega\left(y^{(\nu)}(x)\right) .
$$

Then, by Theorem 1 of [8], either $\omega_{n}(y)=\omega(v)$ or $\omega_{n}(y)$ is greater than $\omega(v)$ and belongs to $\mathcal{X}$. By Lemma 2 (iii) of [9], since $a_{m n} \neq 0$ the set $\mathcal{X}$ has a finite upper bound, $k_{1}$ say, so that in the latter case $\omega_{n}(y) \leqq k_{1}$. Thus the Laplace transforms

$$
Y_{\nu}(s)=\int_{0}^{\infty} y^{(\nu)}(x) e^{-s x} d x, \quad V(s)=\int_{0}^{\infty} v(x) e^{-s x} d x
$$

are regular functions of $s$ for $R(s)>k=\max \left(k_{1}, \omega(v)\right)$.

We use $\delta, \delta_{1}, \delta_{2}, \cdots, \epsilon, \epsilon_{1}, \epsilon_{2}, \cdots, \eta$ to denote positive numbers, to be thought of as small, $\epsilon$ being arbitrary. $M_{1}, M_{2}, \cdots, N, P$ and $r$ are positive integers, $k, k_{1}, k_{2}, k_{3}$ are real numbers and $T$ is a positive number. The numbers $a_{r}=a+i t_{r}$, where $a, t_{r}$ are real, denote zeros of $\tau(s)$ on the line $\mathcal{R}(s)=a$. We define the function

$$
\vartheta(s)=\sum_{\mu=0}^{m} \sum_{\nu=0}^{n} a_{\mu \nu}^{\prime} s^{\nu} e^{b_{\mu} s}
$$

and write

$$
\beta_{r}=\vartheta\left(a_{r}\right) /\left\{\tau^{\prime}\left(a_{r}\right)\right\}=\beta_{r}^{\prime}+i \beta_{r}^{\prime \prime}
$$

if $\tau^{\prime}\left(a_{r}\right) \neq 0$ and 


$$
\gamma_{r}=\frac{2 \vartheta^{\prime}\left(a_{r}\right)}{\tau^{\prime \prime}\left(a_{r}\right)}-\frac{2 \vartheta\left(a_{r}\right) \tau^{\prime \prime \prime}\left(a_{r}\right)}{3\left\{\tau^{\prime \prime}\left(a_{r}\right)\right\}^{2}}=\gamma_{r}^{\prime}+i \gamma_{r}^{\prime \prime}, A_{r}=-\frac{2 \vartheta\left(a_{r}\right)}{\tau^{\prime \prime}\left(a_{r}\right)}
$$

if $\tau^{\prime \prime}\left(a_{r}\right) \neq 0$, where $\beta_{r}^{\prime}, \beta_{r}^{\prime \prime \prime}, \gamma_{r}^{\prime}, \gamma_{r}^{\prime \prime}$ are real.

Using the notation of [10], we write $X_{r}$ for any value of $\left(x A_{r}^{-1}\right)^{1 / 2}$ and $\alpha_{r}=\arg A_{r}, \alpha_{r}^{\prime}=\arg \left(-A_{r}\right)$, where $-\pi<\alpha_{r} \leqq \pi,-\pi \leqq \alpha_{r}^{\prime}<\pi$. We also write $X_{j, r}(j=-1,0$ or 1$), X_{r}^{\prime}, X_{r}^{\prime \prime}$ for the particular values of $X_{r}$ for which $\arg X_{j, r}=-\alpha_{r} / 2-\pi j, \quad \arg X_{r}^{\prime}=-\alpha_{r}^{\prime} / 2-\pi / 2, \quad \arg X_{r}^{\prime \prime}=-\alpha_{r}^{\prime} / 2+\pi / 2$.

Then we define the function

$$
H_{r}\left(X_{r}\right)=(1 / 2)(\pi x)^{-1 / 2} X_{r}^{3 / 2-\gamma_{r}} \exp \left(2 A_{r} X_{r}\right),
$$

where the positive square root of $(\pi x)^{-1}$ is taken.

We use $C, C_{1}, C_{2}, C_{2}^{\prime}, C_{2}^{\prime \prime}, C_{3}, \cdots$ to denote numbers calculable in terms of some or all of the $a_{\mu \nu}, a_{\mu \nu}^{\prime}, b_{\mu}$ and the values of $v(x)$ and of $y^{(\nu)}(x)$ given by the boundary conditions; of these, $C$ is not always the same at each occurrence, and we write $C_{1}\left(a_{r}\right)$ for $C_{1}$, for example, to express the fact that $C_{1}\left(a_{r}\right)$ denotes a certain function of $a_{r}$ and the corresponding numbers $\beta_{r}, \gamma_{r}$, and $A_{r}$. The numbers $K, K_{1}, K_{2}, \cdots$, of which $K$ is not always the same at each occurrence, are positive numbers independent of $x, T$ and $s=\sigma+i t$, but possibly dependent on any of the other parameters mentioned above; the constant implied in the $O($ ) notation is of the type $K . \phi(s)$ denotes a function (not always the same at each occurrence) which has a zero at the origin and is regular for $|s| \leqq K$ for some $K$. We define the set $S$ to be the set of all points $s^{\prime}=\sigma^{\prime}+i t^{\prime}$ satisfying the condition that there exist numbers $K$ such that $\tau(s)$ has no zeros in the semi-infinite strip

$$
\sigma \geqq \sigma^{\prime}-K, \quad t^{\prime}-K \leqq t \leqq t^{\prime}+K .
$$

In the following theorems the notation $O($ ) refers to the passage of $x$ to infinity through positive values.

THEOREM 1. If (i) $\tau(s)$ has a zero of order $\lambda_{r}$ at each of the points $s=a_{r}$ $(r=1,2, \cdots, N)$, where $a_{r}=a+i t_{r} \neq 0$, and has no other zero in the half-plane $\sigma \geqq a-\delta$ and (ii) $\omega(v)<a-\delta$, then for $\nu=0,1, \cdots, n-1$

$$
y^{(\nu)}(x)=\sum_{r=1}^{N} a_{r}^{\nu} e^{a_{r} x} Q\left(a_{r}, x\right),
$$

where $Q\left(a_{r}, x\right)$ is defined as follows:

$$
\begin{array}{rlrl}
\lambda_{r}=1 ; \quad Q\left(a_{r}, x\right) & =x^{-\beta_{r}}\left\{C_{1}\left(a_{r}\right)+O\left(x^{-K}\right)\right\} . & \\
\lambda_{r}=2, \quad \vartheta\left(a_{r}\right) & =0 ; & & \\
Q\left(a_{r}, x\right) & =x^{1-\gamma_{r}}\left\{C_{2}^{\prime}\left(a_{r}\right)+O\left(x^{-K}\right)\right\}+C_{2}^{\prime \prime}\left(a_{r}\right) & & \left(\gamma_{r} \neq 1\right), \\
& =\log x\left\{C_{2}\left(a_{r}\right)+O\left(x^{-K}\right)\right\}+C_{3}\left(a_{r}\right) & & \left(\gamma_{r}=1\right) .
\end{array}
$$


(c)

$$
\begin{aligned}
& \lambda_{r}=2, \quad \vartheta\left(a_{r}\right) \neq 0 ; \\
& Q\left(a_{r}, x\right)=H_{r}\left(X_{r}^{\prime}\right)\left\{C_{4}^{\prime}\left(a_{r}\right)+O\left(x^{-K}\right)\right\}+H_{r}\left(X_{r}^{\prime \prime}\right)\left\{C_{4}^{\prime \prime}\left(a_{r}\right)+O\left(x^{-K}\right)\right\} \\
&=H_{r}\left(X_{0, r}\right)\left\{C_{4}\left(a_{r}\right)+O\left(x^{-K}\right)\right\} \\
&\left(-\pi<\alpha_{r} \leqq \pi\right),
\end{aligned}
$$

THEOREM 2. If $a_{r}=0$ for $r=p$, where $p$ takes any one of the values $1,2, \cdots, N$, and otherwise the conditions of Theorem 1 are satisfied, then

$$
y^{(\nu)}(x)=\sum_{r \neq p} a_{r}^{\nu} e^{a_{r} x} Q\left(a_{r}, x\right)+Q_{\nu}(0, x),
$$

where $Q_{0}(0, x)=Q(0, x)$ and, for $\nu=1,2, \cdots, n-1, Q_{\nu}(0, x)$ is defined in the various cases as follows:

(a) $Q_{\nu}(0, x)=x^{-\beta_{r-\nu}}\left\{(-1)^{\nu} \beta_{r}\left(\beta_{r}+1\right) \cdots\left(\beta_{r}+\nu-1\right) C_{1}(0)+O\left(x^{-K}\right)\right\}$.

(b) $Q_{\nu}(0, x)=x^{1-\gamma_{r}-\nu}\left\{(-1)^{\nu}\left(\gamma_{r}-1\right) \gamma_{r}\left(\gamma_{r}+1\right) \cdots\left(\gamma_{r}+\nu-2\right) C_{2}^{\prime}(0)+O\left(x^{-K}\right)\right\}$

$$
=x^{-\nu}\left\{(-1)^{\nu-1} \Gamma(\nu) C_{2}(0)+O\left(x^{-K}\right)\right\} \quad\left(\gamma_{r}=1\right) .
$$

(c) $\quad Q_{\nu}(0, x)=\left(X_{r}^{\prime}\right)^{-\nu} H_{r}\left(X_{r}^{\prime}\right)\left\{C_{4}^{\prime}(0)+O\left(x^{-K}\right)\right\}$

$$
\begin{aligned}
& +\left(X_{r}^{\prime \prime}\right)^{-\nu} H_{r}\left(X_{r}^{\prime \prime}\right)\left\{C_{4}^{\prime \prime}(0)+O\left(x^{-K}\right)\right\} \\
& \left(-\pi<\alpha_{r} \leqq \pi\right), \\
& =X_{0, r}^{-v} H_{r}\left(X_{0, r}\right)\left\{C_{4}(0)+O\left(x^{-K}\right)\right\} \\
& \left(\alpha_{r} \neq \pi\right) \text {. }
\end{aligned}
$$

THEOREM 3. If $y^{(n)}(x)$ is continuous and of bounded variation for $0 \leqq x \leqq b_{m}$ and the same is true of $v(x)$ in every finite interval $0 \leqq x \leqq x_{0}$, then the results of Theorems 1 and 2 hold for $\nu=n$.

Under certain conditions there may be zeros of $\tau(s)$ in the half-plane $\sigma \geqq a$ which are not singularities of $Y_{\nu}(s)$, and so do not contribute to the asymptotic expansion of $y^{(v)}(x)$. The results of the above theorems hold in this case also.

3. Differential equation for $Y_{0}(s)$. For $\sigma>k$ it follows from (1.1) and (2.1) that $Y_{0}(s)$ satisfies the differential equation

$$
\tau(s) Y_{0}^{\prime}(s)+\left\{\tau^{\prime}(s)-\vartheta(s)\right\} Y_{0}(s)=W(s)-V(s),
$$

where

$$
\begin{aligned}
W(s)= & \sum_{\mu=0}^{m} \sum_{\nu=1}^{n} e^{b_{\mu} s} \sum_{l=0}^{\nu-1}\left\{a_{\mu \nu}(\nu-l-1) s^{\nu-l-2}\right. \\
& \left.+\left(b_{\mu} a_{\mu \nu}-a_{\mu \nu}^{\prime}\right) s^{\nu-l-1}\right\} y^{(l)}(0)-W_{1}(s), \\
W_{1}(s)= & \sum_{\mu=1}^{m} \sum_{\nu=0}^{n} e^{b_{\mu} s} \int_{0}^{b_{\mu}}\left(a_{\mu \nu} x-b_{\mu} a_{\mu \nu}+a_{\mu \nu}^{\prime}\right) y^{(\nu)}(x) e^{-s x} d x .
\end{aligned}
$$

The boundary conditions on $y^{(\nu)}(x)$ imply $Y_{0}(s)=s^{-1}\{y(0)+o(1)\}$ as $\sigma \rightarrow \infty$ 
for fixed $t$, and, if $W(s)-V(s)$ is not identically zero $\left({ }^{3}\right)$, the solution of (3.1) satisfying this condition is

$$
\begin{aligned}
Y_{0}(s)= & \frac{1}{\tau(s)} \exp \left(\int_{s_{0}}^{s} \frac{\vartheta(\xi)}{\tau(\xi)} d \xi\right) \int_{s+\infty}^{s}\{W(\zeta)-V(\zeta)\} \\
& \cdot \exp \left(-\int_{s_{0}}^{\zeta} \frac{\vartheta(\xi)}{\tau(\xi)} d \xi\right) d \zeta .
\end{aligned}
$$

Here $s+\infty$ denotes the point at infinity in the positive direction on the parallel through $s$ to the real axis, the path of integration is a straight line, and $s_{0}$ is an arbitrary fixed point in the half-plane $\sigma>k$. The integral converges if $R\left(C_{5}\right)<0$, where

$$
C_{5}=b_{m}-a_{m n}^{\prime} / a_{m n}
$$

and this condition involves no loss of generality. For the transformation $x=x_{1}+K_{1}$, where $K_{1}>R\left(C_{5}\right)$, reduces (1.1) to an equation of similar form in $y\left(x_{1}+K_{1}\right) \equiv y_{1}\left(x_{1}\right)$ for which the required condition is satisfied. The boundary values of $y_{1}^{(\nu)}\left(x_{1}\right)$ in $\left(0, b_{m}\right)$ may be determined from those of $y^{(\nu)}(x)$ in a finite number of stages, by the solution of an integral equation for $y^{(n)}\left(x+b_{m}\right)$. We may therefore take

$$
\left|\arg \left(-C_{5}\right)\right|<\pi / 2 \text {. }
$$

Since $\tau(s), \vartheta(s)$, and $W(s)$ are integral functions, (3.2) provides the analytic continuation of $Y_{0}(s)$ for all points of the half-plane $\sigma>\omega(v)$ which belong to $S, s_{0}$ being an arbitrary point satisfying the same conditions. We now write

$$
y_{2}(x)=y(x) e^{-a_{r} x}, \quad v_{2}(x)=v(x) e^{-a_{r} x}, \quad Y_{02}(s)=Y_{0}\left(s+a_{r}\right)
$$

and denote $\tau\left(s+a_{r}\right), \vartheta\left(s+a_{r}\right), W\left(s+a_{r}\right), V\left(s+a_{r}\right)$ by $\tau_{2}(s), \vartheta_{2}(s), W_{2}(s), V_{2}(s)$ respectively. Then the solution of the equation for $Y_{02}(s)$ corresponding to (3.1) is

$$
\begin{aligned}
Y_{02}(s)= & \frac{1}{\tau_{2}(s)} \exp \left(\int_{s_{0}}^{s} \frac{\vartheta_{2}(\xi)}{\tau_{2}(\xi)} d \xi\right) \int_{s+\infty}^{s}\left\{W_{2}(\zeta)-V_{2}(\zeta)\right\} \\
& \cdot \exp \left(-\int_{s_{0}}^{\zeta} \frac{\vartheta_{2}(\xi)}{\tau_{2}(\xi)} d \xi\right) d \zeta
\end{aligned}
$$

for all points of the half-plane $\sigma>\omega(v)-a$ which belong to $\mathcal{S}, s_{0}$ being an arbitrary point satisfying the same conditions. Also, by repeated integration by parts we have

(3) If $W(s)-V(s) \equiv 0$, the solution of (3.1) is $Y_{0}(s)=D\{\tau(s)\}^{-1} \exp \left(\int \vartheta(s)\{\tau(s)\}^{-1} d s\right)$, where $D$ is an arbitrary complex number. Then the coefficients in the results of Theorems 1 and 2 are replaced by arbitrary numbers. 


$$
Y_{\nu}\left(s+a_{r}\right)=\left(s+a_{r}\right)^{\nu} Y_{02}(s)-\sum_{l=0}^{v-1}\left(s+a_{r}\right)^{\nu-l-1} y^{(l)}(0),
$$

so that an expression for $Y_{\nu}(s)$ in the neighborhood of a zero $s=a_{r}$ of $\tau(s)$ may be determined by considering the behavior of $Y_{02}(s)$, as given by (3.4), in the neighborhood of the origin.

4. Simple zero of $\tau(s)$. We suppose first that $\tau(s)$ has a simple zero at the point $s=a_{r}=a+i t_{r}$ and that there exists a positive number $\delta_{r} \leqq \delta$ such that $\tau(s)$ has no other zero in the strip $\sigma \geqq a-\delta_{r},\left|t-t_{r}\right| \leqq \delta_{r}$, and also that $\omega(v)$ $<a-\delta$. Then $\tau_{2}(s)$ has a simple zero at the origin and no other zero in the strip $\sigma \geqq-\delta_{r},|t| \leqq \delta_{r}$, and $V_{2}(s)$ is regular for $\sigma \geqq-\delta$, while the functions $\phi(s)$ which arise in this paragraph and in $\$ 5$ are regular for $|s| \leqq \delta_{r}$.

Since $\vartheta_{2}(0) /\left\{\tau_{2}^{\prime}(0)\right\}=\beta_{r}$, the integrand in (3.4) is of the order of $\zeta^{-\beta_{r}}$ at the origin. Thus if $R\left(\beta_{r}\right)=\beta_{r}^{\prime}<1$ the path of integration from $s+\infty$ to $s$ may be taken to consist of the two straight lines from $\infty$ to 0 and from 0 to $s$, since for real values of $R$

$$
\int_{R}^{R+i t}\{W(\zeta)-V(\zeta)\} \exp \left(-\int_{s_{0}}^{\zeta} \frac{\vartheta(\xi)}{\tau(\xi)} d \xi\right) d \zeta \rightarrow 0
$$

as $R \rightarrow \infty$ for all $t$. Hence, for $0<|s| \leqq \delta_{r},|\arg s| \leqq \pi / 2+\epsilon_{r}$, where $\epsilon_{r}$ is taken to be less than $\pi / 4$, we have

$$
Y_{02}(s)=C_{6}\left(a_{r}\right) s^{\beta_{r}-1}\{1+\phi(s)\}+C_{7}\left(a_{r}\right)+\phi(s) .
$$

Here

$$
\begin{aligned}
C_{6}\left(a_{r}\right) & =\frac{1}{\tau^{\prime}\left(a_{r}\right)} \int_{a_{r}+\infty}^{a_{r}} G_{1}\left(\zeta, a_{r}\right) d \zeta, \\
G_{1}\left(\zeta, a_{r}\right) & =\{W(\zeta)-V(\zeta)\}\left(\zeta-a_{r}\right)^{-\beta_{r}} \exp \left\{-\int_{a_{r}}^{\zeta}\left(\frac{\vartheta(\xi)}{\tau(\xi)}-\frac{\beta_{r}}{\xi-a_{r}}\right) d \xi\right\}, \\
C_{7}\left(a_{r}\right) & =\left\{W\left(a_{r}\right)-V\left(a_{r}\right)\right\} /\left\{\left(1-\beta_{r}\right) \tau^{\prime}\left(a_{r}\right)\right\} .
\end{aligned}
$$

If $M_{r}<\beta_{r}^{\prime}<M_{r}+1$ for some positive integer $M_{r}$, for $0<|\zeta| \leqq \delta_{r}$ the integrand in (3.4) may be expressed in the form

$$
\sum_{l=0}^{M_{r}-1} d_{l}\left(a_{r}\right) \zeta^{l-\beta_{r}}+\zeta^{M_{r}-\beta_{r}}\{C+\phi(\zeta)\}
$$

where the coefficients $d_{l}$ are of the type $C$. The integral from $s+\infty$ to $s$ of the first part of this expression is convergent, and the second part is integrable up to the origin. Thus (4.2) holds with $C_{6}\left(a_{r}\right)$ given by

$$
C_{6}\left(a_{r}\right)=\frac{1}{\tau^{\prime}\left(a_{r}\right)} \int_{a_{r}+\infty}^{a_{r}}\left\{G_{1}\left(\zeta, a_{r}\right)-\sum_{l=0}^{M_{r}-1} d_{l}\left(a_{r}\right)\left(\zeta-a_{r}\right)^{l-\beta_{r}}\right\} d \zeta .
$$


If $\beta_{r}^{\prime}=M_{r}$ and $\Im\left(\beta_{r}\right)=\beta_{r}^{\prime \prime} \neq 0$, for $0<|\zeta| \leqq \delta_{r}$ the integrand in (3.4) may be expressed in the form

$$
\exp \left(C_{5} \zeta\right) \sum_{l=0}^{M_{r}-1} g_{l}\left(a_{r}\right) \zeta^{l-\beta_{r}}+\zeta^{i \beta^{\prime \prime}}\{C+\phi(\zeta)\},
$$

where the coefficients $g_{l}$ are of the type $C$. Here the factor $\exp \left(C_{b} \zeta\right)$ is introduced to ensure that the integral from $s+\infty$ to $s$ of the first part of the expression is convergent, using (3.3). By repeated integration by parts we obtain (4.2) with

$$
\begin{aligned}
C_{6}\left(a_{r}\right)= & \frac{1}{\tau^{\prime}\left(a_{r}\right)} \int_{a_{r}+\infty}^{a_{r}}\left\{G_{1}\left(\zeta, a_{r}\right)-\exp \left\{C_{5}\left(\zeta-a_{r}\right)\right\} \sum_{l=0}^{M_{r}-1} g_{l}\left(a_{r}\right)\left(\zeta-a_{r}\right)^{l-\beta_{r}}\right\} d \zeta \\
& -\frac{1}{\tau^{\prime}\left(a_{r}\right)} \sum_{l=0}^{M_{r}-1} g_{l}\left(a_{r}\right)\left(-C_{5}\right)^{\beta_{r}-l-1} \Gamma\left(1+l-\beta_{r}\right) .
\end{aligned}
$$

If $\beta_{r}=M_{r}$, a term of the form $\zeta^{-1} \exp \left(C_{5} \zeta\right)$ arises in the integrand of (3.4), and (4.2) is replaced by

$$
Y_{02}(s)=C_{8}\left(a_{r}\right) s^{M_{r}-1} \log s\{1+\phi(s)\}+C_{9}\left(a_{r}\right)+\phi(s),
$$

where

$$
\begin{aligned}
C_{8}\left(a_{r}\right)= & \frac{C_{5}^{M_{r}-1}}{\tau^{\prime}\left(a_{r}\right)}\left\{\frac{W\left(a_{r}\right)-V\left(a_{r}\right)}{\Gamma\left(M_{r}\right)}+\sum_{l=1}^{M_{r}-1} g_{l}\left(a_{r}\right) \frac{C_{5}^{-l}}{\Gamma\left(M_{r}-l\right)}\right\}, \\
C_{9}\left(a_{r}\right)= & \left\{W\left(a_{r}\right)-V\left(a_{r}\right)\right\} /\left\{\left(1-M_{r}\right) \tau^{\prime}\left(a_{r}\right)\right\} \quad \\
= & \left\{\tau^{\prime}\left(a_{r}\right)\right\}-1\left\{W\left(a_{r}\right)-V\left(a_{r}\right)\right\}\left\{\log \left(-C_{5}\right)-\Gamma^{\prime}(1)\right\}+C_{6}^{\prime}\left(a_{r}\right), \\
C_{6}^{\prime}\left(a_{r}\right)= & \frac{1}{\tau^{\prime}\left(a_{r}\right)} \int_{a_{r}+\infty}^{a_{r}}\left\{G_{1}\left(\zeta, a_{r}\right)-\left\{W\left(a_{r}\right)-V\left(a_{r}\right)\right\}\right.
\end{aligned}
$$

5. Double zero of $\tau(s)$. We now assume that the zero of $\tau(s)$ at $s=a_{r}$ is of order two and that the other conditions are the same as in $\S 4$. Then if $\vartheta\left(a_{r}\right)=0$ the integrand in (3.4) is of the order of $\zeta^{-\gamma_{r}}$ at the origin, and if $\gamma_{r}$ is not a positive integer $Y_{02}(s)$ is given by

$$
Y_{02}(s)=C_{10}\left(a_{r}\right) s^{\gamma_{r}-2}\{1+\phi(s)\}+C_{11}\left(a_{r}\right) s^{-1}+C+\phi(s) .
$$

Here $C_{10}\left(a_{r}\right), C_{11}\left(a_{r}\right)$ are equal to $C_{6}\left(a_{r}\right), C_{7}\left(a_{r}\right)$ respectively with $\tau^{\prime}\left(a_{r}\right), \beta_{r}$ replaced by $\tau^{\prime \prime}\left(a_{r}\right) / 2, \gamma_{r}$ respectively. If $\gamma_{r}=M_{r}$ we have

$$
Y_{02}(s)=C_{12}\left(a_{r}\right) s^{M_{r}-2} \log s\{1+\phi(s)\}+C_{13}\left(a_{r}\right) s^{-1}+C+\phi(s),
$$

where $C_{12}\left(a_{r}\right), C_{13}\left(a_{r}\right)$ are equal to $C_{8}\left(a_{r}\right), C_{9}\left(a_{r}\right)$ respectively with the above replacements. 
In the general case where $\vartheta\left(a_{r}\right) \neq 0$, for points $\zeta, s$ and $s_{0}$ in the annulus $0<|\zeta| \leqq \delta_{r}$ we have

$$
\begin{aligned}
& \exp \left(\int_{s_{0}}^{s} \frac{\vartheta_{2}(\xi)}{\tau_{2}(\xi)} d \xi-\int_{s_{0}}^{\zeta} \frac{\vartheta_{2}(\xi)}{\tau_{2}(\xi)} d \xi\right) \\
& =s^{\gamma_{r}} \exp \left\{A_{r} s^{-1}+\phi(s)\right\} \zeta^{-\gamma_{r}} \exp \left\{-\frac{A_{r}}{\zeta}-\int_{0}^{\zeta}\left(\frac{\vartheta_{2}(\xi)}{\tau_{2}(\xi)}+\frac{A_{r}}{\xi^{2}}-\frac{\gamma_{r}}{\xi}\right) d \xi\right\},
\end{aligned}
$$

and this function is integrable up to the origin along a path on which $R\left(A_{r} \zeta^{-1}\right)>0$. We suppose the $\zeta$-plane to be cut along the line $\arg \zeta=\pi$ and take $z$ to be a point on the circle $|\zeta|=|s|$ in this cut plane, with $R\left(A_{r} z^{-1}\right)>0$. We denote by $\mathcal{C}(z, s)$ the arc of this circle joining the points $z$ and $s$, and by $\mathcal{B}(z)$ any simple path in the cut plane which joins the point at infinity on the positive real axis to the point $z$, without passing through the origin.

Using repeated integration by parts, by (3.4), (4.1), and (5.3) we have

$$
Y_{02}(s)=s^{\gamma_{r}-2} \exp \left(A_{r} s^{-1}\right)\{1+\phi(s)\}\left\{C_{14}^{(\psi)}\left(a_{r}\right)+F(z, s)\right\}+C+\phi(s),
$$

where $\psi=\arg z$, with $\cos \left(\alpha_{r}-\psi\right)>0$, and

$$
\begin{aligned}
C_{14}^{(\psi)}\left(a_{r}\right)= & \frac{2}{\tau_{2}^{\prime \prime}(0)}\left(\int_{\mathcal{B}_{(z)}}+\int_{z}^{0}\right)\left\{W_{2}(\zeta)-V_{2}(\zeta)\right\} \zeta^{-\gamma_{r}} \\
& \cdot \exp \left\{-\frac{A_{r}}{\zeta}-\int_{0}^{\zeta}\left(\frac{\vartheta_{2}(\xi)}{\tau_{2}(\xi)}+\frac{A_{r}}{\xi^{2}}-\frac{\gamma_{r}}{\xi}\right) d \xi\right\} d \zeta \\
= & \frac{2}{\tau^{\prime \prime}\left(a_{r}\right)} \int_{a_{r}+\infty}^{a_{r}+0 \exp (i \psi)} G_{2}\left(\zeta, a_{r}\right) d \zeta, \\
G_{2}\left(\zeta, a_{r}\right)= & \{W(\zeta)-V(\zeta)\}\left(\zeta-a_{r}\right)^{-\gamma_{r}} \\
& \cdot \exp \left\{-\frac{A_{r}}{\zeta-a_{r}}-\int_{a_{r}}^{\zeta}\left(\frac{\vartheta(\xi)}{\tau(\xi)}+\frac{A_{r}}{\left(\xi-a_{r}\right)^{2}}-\frac{\gamma_{r}}{\xi-a_{r}}\right) d \xi\right\}, \\
F(z, s)= & \frac{2}{\tau^{\prime \prime}\left(a_{r}\right)}\left(\int_{0}^{z}+\int_{\mathcal{C}_{(z, s)}}\right) \phi(\zeta) \zeta^{P-\gamma_{r}} \\
& \cdot \exp \left(-A_{r} \zeta^{-1}\right) d \zeta=F_{1}(z)+F_{2}(z, s) .
\end{aligned}
$$

Here the path of integration for $C_{14}^{(\psi)}\left(a_{r}\right)$ does not cross the line arg $\left(\zeta-a_{r}\right)=\pi$, while that from 0 to $z$ is a straight line, and the positive integer $P$ may be arbitrarily large.

For $\alpha_{r}= \pm \pi / 2$ a modification of these results is required, and here the path of integration in (3.4) is rotated through an angle $\mp \eta$, where $0<\eta$ $<\min \left\{\pi / 4, \pi / 2-\left|\arg \left(-C_{5}\right)\right|\right\}$, and the $\zeta$-plane is assumed to be cut along the line $\arg \zeta=\pi-\eta$ if the rotation is negative, and along the line $\arg \zeta$ $=\pi+\eta$ if the rotation is positive. Then $(5.5)$ for $C_{14}^{(\psi)}\left(a_{r}\right)$ is replaced by 


$$
C_{14}^{(\psi)}\left(a_{r}\right)=\frac{2}{\tau^{\prime \prime}\left(a_{r}\right)} \int_{a_{r}+\infty \exp (-i \eta)}^{a_{r}+0 \exp (i \psi)} G_{2}\left(\zeta, a_{r}\right) d \zeta
$$

where the path of integration does not cross the line arg $\left(\zeta-a_{r}\right)=\pi-\eta$, or by

$$
C_{14}^{(\psi)}\left(a_{r}\right)=\frac{2}{\tau^{\prime \prime}\left(a_{r}\right)} \int_{a_{r}+\infty \exp (i \eta)}^{a_{r}+0 \exp (i \psi)} G_{2}\left(\zeta, a_{r}\right) d \zeta,
$$

where the path of integration does not cross the line arg $\left(\zeta-a_{r}\right)=\pi+\eta$.

We now establish three lemmas concerning $F(z, s)$ considered as a function of $s$, using the notation $O()$ with reference to the passage of $|s|$ to zero, and writing $\theta=\alpha_{r}-\arg s$.

Lemma 1. If $R\left(A_{r} z^{-1}\right) \geqq \mathcal{R}\left(A_{r} s^{-1}\right)$, then

$$
F_{1}(z) \equiv \frac{2}{\tau^{\prime \prime}\left(a_{r}\right)} \int_{0}^{z} \phi(\zeta) \zeta^{P-\gamma_{r}} \exp \left(-A_{r} \zeta^{-1}\right) d \zeta=O\left\{s^{P+2-\gamma_{r}} \exp \left(-A_{r} s^{-1}\right)\right\}
$$

For, since $|\phi(\zeta)|<K|\zeta|$ for $|\zeta| \leqq \delta_{r}$ and $\cos \left(\alpha_{r}-\psi\right)>0$,

$$
F_{1}(z)=O\left\{z^{P+2-\gamma_{r}} \exp \left(-A_{r} z^{-1}\right)\right\}=O\left\{s^{P+2-\gamma_{r}} \exp \left(-A_{r} s^{-1}\right)\right\} .
$$

LEMMA 2. If either

(i) $\psi>\arg s$ and $\cos (\theta-v) \geqq \cos \theta$ for $0 \leqq v \leqq \psi-\arg s$ or (ii) $\psi<\arg s$ and $\cos (\theta+v) \geqq \cos \theta$ for $0 \leqq v \leqq \arg s-\psi$, then

$$
F_{2}(z, s) \equiv \frac{2}{\tau^{\prime \prime}\left(a_{r}\right)} \int_{C_{(z, 8)}} \phi(\zeta) \zeta^{P-\gamma_{r}} \exp \left(-A_{r} \zeta^{-1}\right) d \zeta=O\left\{s^{P+2-\gamma_{r}} \exp \left(-A_{r} s^{-1}\right)\right\}
$$

This follows directly by an elementary calculation.

LemMA 3. For all values of $\alpha_{r}$ and $\arg s$ such that $|\arg s| \leqq \pi / 2+\epsilon_{r}$,

$$
F(z, s) \equiv F_{1}(z)+F_{2}(z, s)=O\left\{s^{P+2-\gamma_{r}} \exp \left(-A_{r} s^{-1}\right)\right\} .
$$

In the proof of this result we consider separately the different values of $\alpha_{r}$ for which the range of variation of arg $z$ must be differently defined. The positive numbers $\epsilon, \epsilon_{r}$, where $\epsilon$ is arbitrarily small, are chosen so that $\epsilon<\epsilon_{r}$ and $\epsilon_{r}$ is less than the least positive number of the set $\eta / 2,\left|\alpha_{r}\right| / 2, \pi / 4-\left|\alpha_{r}\right| / 2$, $\left|\alpha_{r}\right| / 3-\pi / 6$, and $\pi-\left|\alpha_{r}\right|$.

(i) $\left|\alpha_{r}\right|<\pi / 2$. Here the condition $\cos \left(\alpha_{r}-\psi\right)>0$ is satisfied by

$$
\alpha_{r}-\pi / 2+\epsilon \leqq \psi \leqq \alpha_{r}+\pi / 2-\epsilon
$$

for all values of $\arg s$.

(a) For $\alpha_{r}-\pi / 2+\epsilon \leqq \arg s \leqq \alpha_{r}+\pi / 2-\epsilon$, (5.7) is satisfied for $z=s$, and the contour $\mathcal{C}(z, s)$ reduces to a single point. Thus the lemma follows from Lemma 1.

(b) The range $\alpha_{r}+\pi / 2-\epsilon<\arg s \leqq \pi / 2+\epsilon_{r}$ occurs only for $\alpha_{r} \leqq 0$, and 
here (5.7) is satisfied for $\psi=\arg s-\pi / 2$, and Lemma 1 again holds. Also condition (ii) of Lemma 2 is satisfied, so that Lemma 3 is proved. For the range $-\pi / 2-\epsilon_{r} \leqq \arg s<\alpha_{r}-\pi / 2+\epsilon$, which occurs only for $\alpha_{r} \geqq 0$, we take $\psi=\arg s+\pi / 2$ and the result follows similarly, using condition (i) of Lemma 2 .

(ii) $\pi / 2<\alpha_{r} \leqq \pi$. Here the sector $R\left(A_{r} \zeta^{-1}\right)>0$ is divided into two parts by the line $\arg \zeta=\pi$, so that we require either

$$
\alpha_{r}-\pi / 2+\epsilon \leqq \psi \leqq \pi
$$

or

$$
-\pi \leqq \psi \leqq \alpha_{r}-3 \pi / 2-\epsilon .
$$

(a) For $\alpha_{r}-\pi / 2+\epsilon \leqq \arg s \leqq \pi / 2+\epsilon_{r}$, (5.8a) is satisfied for $z=s$ and the lemma is proved as in (i) (a).

(b) For $\alpha_{r}-\pi / 2-\epsilon \leqq \arg s<\alpha_{r}-\pi / 2+\epsilon$ with $\psi=\arg s+2 \epsilon$, and for $\alpha_{r}-\pi \leqq \arg s<\alpha_{r}-\pi / 2-\epsilon$ with $\psi=\arg s+\pi / 2+\epsilon$, (5.8a) and condition (i) of Lemma 2 are satisfied, so that in each case Lemma 3 is proved.

(c) For $\alpha_{r}-\pi-2 \epsilon_{r} \leqq \arg s \leqq \alpha_{r}-\pi$ with $\psi=\arg s-\pi / 2-\epsilon$, and for $-\pi / 2-\epsilon_{r} \leqq \arg s<\alpha_{r}-\pi-2 \epsilon_{r}$ with $\psi=\arg s-\pi / 2+\epsilon_{r}$, (5.8b) and condition (ii) of Lemma 2 are satisfied, so that Lemma 3 again follows.

(iii) $-\pi<\alpha_{r}<-\pi / 2$. The conditions required here are either

$$
\alpha_{r}+3 \pi / 2+\epsilon \leqq \psi \leqq \pi
$$

or

$$
-\pi \leqq \psi \leqq \alpha_{r}+\pi / 2-\epsilon,
$$

and the proof of the lemma is similar to that of case (ii).

(iv) $\alpha_{r}=\pi / 2$. Here we consider $z$ and $\mathcal{C}(z, s)$ to lie in the plane cut along the line arg $\zeta=\pi-\eta$, and require either

$$
\epsilon \leqq \psi \leqq \pi-\eta
$$

or

$$
-\pi-\eta \leqq \psi \leqq-\pi-\epsilon .
$$

(a) For $\epsilon \leqq \arg s \leqq \pi / 2+\epsilon_{r}$, (5.10a) is satisfied for $z=s$ and the lemma is proved as in (i) (a).

(b) For $-\epsilon \leqq \arg s<\epsilon$ with $\psi=\arg s+2 \epsilon$, and for $-\pi / 2 \leqq \arg s<-\epsilon$ with $\psi=\arg s+\pi / 2+\epsilon,(5.10 \mathrm{a})$ and condition (i) of Lemma 2 are satisfied, and Lemma 3 follows as before.

(c) For $-\pi / 2-\epsilon_{r} \leqq \arg s \leqq-\pi / 2$ with $\psi=\arg s-\pi / 2-\epsilon$, (5.10b) and condition (ii) of Lemma 2 are satisfied, so that Lemma 3 again follows.

(v) $\alpha_{r}=-\pi / 2$. Here we take $z$ and $\mathcal{C}(z, s)$ to lie in the plane cut along the line $\arg \zeta=\pi+\eta$, and require either

$$
\pi+\epsilon \leqq \psi \leqq \pi+\eta
$$


or

$$
-\pi+\eta \leqq \psi \leqq-\epsilon .
$$

The proof of the lemma is similar to that of case (iv).

The whole range $-\pi<\alpha_{r} \leqq \pi$ is covered by cases (i)-(v), so that the proof of Lemma 3 is now complete.

The different conditions (5.7)-(5.11) on $\psi$ are satisfied by taking $\psi=\psi_{1}$ or $\psi=\psi_{2}$, where $\psi_{1}, \psi_{2}$ have any values in the following ranges:

$$
\alpha_{r}-\pi / 2+\epsilon \leqq\left\{\begin{array}{l}
\psi_{1} \\
\psi_{2}
\end{array}\right\} \leqq \alpha_{r}+\pi / 2-\epsilon \quad\left(\left|\alpha_{r}\right|<\pi / 2\right),
$$

$\alpha_{r}-\pi / 2+\epsilon \leqq \psi_{1} \leqq \pi, \quad-\pi \leqq \psi_{2} \leqq \alpha_{r}-3 \pi / 2-\epsilon \quad\left(\pi / 2<\alpha_{r} \leqq \pi\right)$,

$\alpha_{r}+3 \pi / 2+\epsilon \leqq \psi_{1} \leqq \pi, \quad-\pi \leqq \psi_{2} \leqq \alpha_{r}+\pi / 2-\epsilon \quad\left(-\pi<\alpha_{r}<-\pi / 2\right)$,

$\epsilon \leqq \psi_{1} \leqq \pi-\eta$,

$-\pi-\eta \leqq \psi_{2} \leqq-\pi-\epsilon$

$$
\left(\alpha_{r}=\pi / 2\right) \text {, }
$$

$\pi+\epsilon \leqq \psi_{1} \leqq \pi+\eta$,

$-\pi+\eta \leqq \psi_{2} \leqq-\epsilon$

$\left(\alpha_{r}=-\pi / 2\right)$.

Then, by Lemma 3, (5.4) may be replaced by

$$
Y_{02}(s)=C_{14}^{(\psi)}\left(a_{r}\right) s^{\gamma_{r}-2} \exp \left(A_{r} s^{-1}\right)\{1+\phi(s)\}+\Omega(s)+C+\phi(s),
$$

where the function $\Omega(s)$ is regular for $0<|s| \leqq \delta_{r},|\arg s| \leqq \pi / 2+\epsilon_{r}$ and satisfies the condition

$$
\Omega(s)=O\left(s^{P}\right)
$$

as $s \rightarrow 0$ within this sector. Here $C_{14}^{(\psi)}\left(a_{r}\right)$ is given by (5.5) if $\left|\alpha_{r}\right| \neq \pi / 2$ and by (5.6a), (5.6b) if $\alpha_{r}=\pi / 2,-\pi / 2$ respectively, and

$$
\begin{aligned}
& \psi=\psi_{1} \text { for } \quad \alpha_{r} \mp \pi \leqq \arg s \leqq \pi / 2+\epsilon_{r}, \\
& \psi=\psi_{2} \text { for } \quad-\pi / 2-\epsilon_{r} \leqq \arg s \leqq \alpha_{r} \mp \pi,
\end{aligned}
$$

the negative sign being taken for $\alpha_{r} \geqq 0$ and the positive sign for $\alpha_{r}<0$. For $\left|\alpha_{r}\right|<\pi / 2$ only one of the values $\psi=\psi_{1}, \psi=\psi_{2}$ is concerned for the whole range $|\arg s| \leqq \pi / 2+\epsilon_{r}$, since $\epsilon_{r}<\pi / 2-\left|\alpha_{r}\right|$, and (5.15) may be replaced by

$$
\begin{aligned}
& \psi=\psi_{1} \text { for } 0 \leqq \alpha_{r}<\pi / 2, \\
& \psi=\psi_{2} \text { for }-\pi / 2<\alpha_{r}<0 .
\end{aligned}
$$

The coefficient $C_{14}^{(\psi)}\left(a_{r}\right)$ is independent of the particular value of $\psi_{1}$ or $\psi_{2}$ taken. For if $\theta_{1}, \theta_{2}$ are any two values of $\psi_{1}$ (say) satisfying any one of the conditions (5.12), then $C_{14}^{\left(\theta_{1}\right)}\left(a_{r}\right)=C_{14}^{\left(\theta_{2}\right)}\left(a_{r}\right)$ provided

$$
\lim _{\rho \rightarrow 0+}\left[\rho^{1-\gamma_{r}} \int_{\theta_{1}}^{\theta_{2}} \exp \left\{-\left|A_{r}\right| \rho^{-1} \cos \left(\alpha_{r}-u\right)\right\} d u\right]=0 .
$$


But $\cos \left(\alpha_{r}-u\right) \geqq \cos (\pi / 2-\epsilon)>0$ for all values of $u$ in the range of integration, so that the result follows.

6. Behavior of $Y_{\nu}(s)$ as $|t| \rightarrow \infty$. We first take $\sigma>k$, and by repeated integration by parts we obtain from (2.1)

$$
s^{\nu} Y_{0}(s)=Y_{\nu}(s)+\sum_{l=0}^{\nu-1} s^{\nu-l-1} y^{(l)}(0) .
$$

Using this in (3.1) it follows that

$$
\tau(s) Y_{\nu}^{\prime}(s)+\left\{\tau^{\prime}(s)-\vartheta(s)-\nu s^{-1} \tau(s)\right\} Y_{\nu}(s)=s^{\nu}\left\{W(s)-V(s)-Z_{\nu}(s)\right\},
$$

where

$$
Z_{\nu}(s)=\sum_{\mu=0}^{m} e^{b_{\mu} \delta} \sum_{p=0}^{n} \sum_{l=0}^{\nu-1}\left\{a_{\mu p}(p-l-1) s^{p-l-2}+\left(b_{\mu} a_{\mu p}-a_{\mu p}^{\prime}\right) s^{p-l-1}\right\} y^{(l)}(0) .
$$

In the remainder of this paragraph and in $\S 7$ we take $\nu=0,1, \cdots, n-1$, and consider $\nu=n$ separately in the proof of Theorem 3. The solution of (6.1) which satisfies the order condition $Y_{\nu}(s)=s^{-1}\left\{y^{(\nu)}(0)+o(1)\right\}$ as $\sigma \rightarrow \infty$ for fixed $t$ is

$$
Y_{\nu}(s)=\frac{s^{\nu}}{\tau(s)} \exp \left(\int_{s_{0}}^{s} \frac{\vartheta(\xi)}{\tau(\xi)} d \xi\right) \int_{s+\infty}^{s}\left\{W(\zeta)-V(\zeta)-Z_{\nu}(\zeta)\right\}
$$

$$
\cdot \exp \left(-\int_{s_{0}}^{\zeta} \frac{\vartheta(\xi)}{\tau(\xi)} d \xi\right) d \zeta
$$

and this expression provides the analytic continuation of $Y_{\nu}(s)$ for all points of the half-plane $\sigma>\omega(v)$ which belong to $\mathcal{S}, s_{0}$ being an arbitrary point satisfying the same conditions.

We suppose that $|t| \rightarrow \infty$ through a set of values for which the corresponding points $s=\sigma+i$ of a strip $\sigma_{1} \leqq \sigma \leqq \sigma_{2}$ belong to $S$, and use the notations $O(), o()$ in this sense, the results holding uniformly with respect to $\sigma$ in the strip. We take $k_{2}>k$ and $\omega(\dot{v})<\sigma_{1}<\sigma_{2} \leqq k_{2}$, and we write $z_{2}=k_{2}+i t$, so that for each value of $t$ the points $z_{2}, s$ lie on a line parallel to the real axis. Then $Y_{\nu}(s)$ may be related to $Y_{\nu}\left(z_{2}\right)$ by considering the path of integration in (6.2) to be made up of two straight lines with the point $z_{2}$ in common.

Using the fact that $y^{(v)}(x)$ has an integrable derivative in every finite interval $\left(0, x_{0}\right)$, we obtain from the definition $(2.1)$ the result

$$
Y_{\nu}\left(z_{2}\right)=o(1)
$$

as $|t| \rightarrow \infty$. Introducing an absolutely continuous function $v_{3}(x)$ such that, for any $x_{2}>0, \int_{0}^{x_{2}}\left|v(x)-v_{3}(x)\right| d x$ is arbitrarily small (see [5, p. 376]), and applying Lemma 2 (iv) of [9] to $\tau(s)$, it follows by (6.2) and (6.3) that

$$
Y_{\nu}(s)=o(1)
$$


as $|t| \rightarrow \infty$ while $s$ belongs to $\mathcal{S}$, uniformly in the strip $\sigma_{1} \leqq \sigma \leqq \sigma_{2}$.

For any fixed number $k_{3}$ such that $\sigma_{1} \leqq k_{3} \leqq \sigma_{2}$ it may also be proved, by a somewhat more detailed discussion of the integral in (6.2), that

$$
Y_{\nu}\left(k_{3}+i t\right)=t^{-1}\left\{C+O\left(t^{-1}\right)\right\}
$$

as $|t| \rightarrow \infty$ while $k_{3}+i t$ belongs to $S$.

7. Inversion of transform $Y_{\nu}(s)$. Since $Y_{\nu}(s)$ has an exponential singularity at $s=a_{r}$ if $\tau(s)$ has a double zero at that point with $\vartheta\left(a_{r}\right) \neq 0$, the results given by Doetsch [2, p. 269, Theorem 4] and used by Cooke [1] are not sufficient here. We therefore use Cauchy's theorem to express $y^{(v)}(x)$ in terms of contour integrals whose asymptotic expansions may be deduced from the results of [10] if an exponential singularity occurs.

We suppose that the conditions of Theorem 1 are satisfied, and we take $k_{2}>k$ as in $\S 6$. We write

$$
\xi_{N}=\min _{1 \leqq r \leqq N} \epsilon_{r}, \quad \rho_{N}=\min _{1 \leqq r \leqq N} \delta_{r}, \quad K_{2}=\rho_{N} \sin \xi_{N},
$$

where $\epsilon_{r}, \delta_{r}$ are the positive numbers of $\$ \S 4,5$. Taking $T>\rho_{N}+\left|t_{r}\right|$ for $r=1,2, \cdots, N$, we denote by $\Lambda_{3}(T)$ the contour consisting of those portions of the straight line $\sigma=a-K_{2},|t| \leqq T$ which lie outside the circles $\left|s-a_{r}\right|$ $=\rho_{N}, r=1,2, \cdots, N$. Then $Y_{\nu}(s)$ is regular within and on the contour $\Lambda(T)$ formed by $\Lambda_{3}(T)$ and the curves

$$
\begin{array}{lr}
\Lambda_{1}(T) & \left(\sigma=k_{2},-T \leqq t \leqq T\right), \\
\Lambda_{2}(T) & \left(a-K_{2} \leqq \sigma \leqq k_{2}, t=-T\right), \\
\Lambda_{4}\left(a_{r}\right)(r=1,2, \cdots, N) & \left(\left|s-a_{r}\right|=\rho_{N},\left|\arg \left(s-a_{r}\right)\right| \leqq \pi / 2+\xi_{N}\right), \\
\Lambda_{5}(T) & \left(a-K_{2} \leqq \sigma \leqq k_{2}, t=T\right),
\end{array}
$$

and

$$
\int_{\Lambda(T)} Y_{\nu}(s) e^{s x} d s=0
$$

Since, for $\nu=0,1, \cdots, n-1, y^{(\nu)}(x)$ is continuous and of bounded variation in the neighborhood of the point $x>0$, the inversion formula given by [6, p. 66, Theorem 7.3] applies. Thus, using (6.4) and (6.5), we obtain for $\nu=0,1, \cdots, n-1$

$$
y^{(\nu)}(x)=\frac{1}{2 \pi i} \sum_{r=1}^{N} \int_{\Lambda_{4}\left(a_{r}\right)} Y_{\nu}(s) e^{s x} d s+O\left\{e^{\left(a-K_{2}\right) x}\right\}
$$

as $x \rightarrow \infty$.

8. Evaluation of integral round $\Lambda_{4}\left(a_{r}\right)$. We require a series of lemmas for the functions denoted by 


$$
\begin{aligned}
& I_{l}^{(1)}\left(a_{r}, x\right)=\frac{1}{2 \pi i} \int_{\Lambda_{4}(0)} C_{14}^{(\psi)}\left(a_{r}\right) s^{\gamma_{r}+l-2}\{1+\phi(s)\} \exp \left(s x+A_{r} s^{-1}\right) d s, \\
& I_{l}^{(2)}\left(a_{r}, x\right)=\frac{1}{2 \pi i} \int_{\Lambda_{4}(0)} s^{l} \Omega(s) e^{8 x} d s,
\end{aligned}
$$

$$
I_{l}\left(a_{r}, x\right)=\frac{1}{2 \pi i} \int_{\Lambda_{4}(0)} s^{l} Y_{02}(s) e^{s x} d s,
$$

where $l=0,1, \cdots, n$ and $x$ takes arbitrarily large positive values. As a preliminary to the discussion of $I_{l}^{(1)}\left(a_{r}, x\right)$ we consider the integral $\int s^{\gamma_{r}+l-2} \exp \left(s x+A_{r} s^{-1}\right) d s$ taken along certain steepest-descent curves. This integral is a particular case of that dealt with in [10], and we utilise some of the notation of that paper.

The saddle-points are the points $s=U$, where $U=\left(x^{-1} A_{r}\right)^{1 / 2}=X_{r}^{-1}$, and the steepest-descent curves required are denoted by $\Gamma_{p}(U)(p=4,5,6)$, while their transforms in the $v$-plane under the transformation $s=U v$ are denoted by $\Gamma_{p} . \Gamma_{4}$ starts from the origin in the direction arg $v=-\pi / 2$, goes through $v=1$ making an angle $\pi / 4$ with the positive direction of the real axis and passes to infinity in the upper half of the plane with the straight line $R(v)=2$ as asymptote, while $\Gamma_{5}$ is the reflexion of $\Gamma_{4}$ in the real axis, but is described from infinity to the origin. $\Gamma_{6}$ is an arc of the circle $|v|=1$ on which $|\arg v|$ $\leqq K_{3}<\pi$, and is required only for $\left|\alpha_{r}\right|<\pi / 2$. The value of $K_{3}$ to be chosen varies with $\alpha_{r}$, and we define $\Gamma_{6}$ to be the arc $|v|=1,|\arg v| \leqq C_{15}\left(a_{r}\right)$ $=\left|\alpha_{r}\right| / 2+\pi / 2+\xi_{N}$. We write $U_{j}=U\left(X_{j, r}\right)=X_{j, r}^{-1}(j=-1,0$ or 1$)$.

The following lemma is a consequence of the results of [10].

LEMma 4. If there exists $K_{4}$ such that one of the following sets of conditions is satisfied, viz.

$$
\begin{aligned}
& p=4, \\
& p=5, \\
& p=6,
\end{aligned}
$$

$$
\begin{array}{r}
K_{4}<\arg U_{j}<\pi-K_{4}, \\
-\pi+K_{4}<\arg U_{j}<-K_{4}, \\
-\pi / 2+K_{4}<\arg U_{j}<\pi / 2-K_{4},
\end{array}
$$

then the integrals

$$
\begin{aligned}
& G_{p}\left(U_{j}\right)=\frac{1}{2 \pi i} \int_{\Gamma_{p}\left(U_{j}\right)} s^{\gamma_{r}+l-2} \exp \left(s x+A_{r} s^{-1}\right) d s, \\
& G_{p}^{\prime}\left(U_{j}\right)=\int_{\Gamma_{p}\left(U_{j}\right)}\left|s^{\gamma_{r}+l-1} \exp \left(s x+A_{r} s^{-1}\right)\right||d s|
\end{aligned}
$$

are convergent and

$$
\begin{aligned}
G_{p}\left(U_{j}\right) & =X_{j, r}^{-l} H_{r}\left(X_{j, r}\right)\left\{1+O\left(x^{-K}\right)\right\}, \\
G_{p}^{\prime}\left(U_{j}\right) & =X_{j, r}^{-l} H_{r}\left(X_{j, r}\right) O\left(x^{-1 / 2}\right) .
\end{aligned}
$$


Lemma 5. For different values of $\alpha_{r}, I_{l}^{(1)}\left(a_{r}, x\right)$ is given by the following expressions:

$$
0 \leqq \alpha_{r}<\pi / 2, \quad X_{0, r}^{-l} H_{r}\left(X_{0, r}\right)\left\{C_{14}^{\left(\psi_{1}\right)}\left(a_{r}\right)+O\left(x^{-K}\right)\right\},
$$

(ii) $-\pi / 2<\alpha_{r}<0$,

$$
X_{0, r}^{-l} H_{r}\left(X_{0, r}\right)\left\{C_{14}^{\left(\psi_{2}\right)}\left(a_{r}\right)+O\left(x^{-K}\right)\right\} \text {, }
$$

(iii) $\pi / 2 \leqq \alpha_{r} \leqq \pi$,

$$
X_{0, r}^{-l} H_{r}\left(X_{0, r}\right)\left\{C_{14}^{\left(\psi_{1}\right)}\left(a_{r}\right)+O\left(x^{-K}\right)\right\}
$$

$$
+X_{-1, r}^{-l} H_{r}\left(X_{-1, r}\right)\left\{C_{14}^{\left(\psi_{2}\right)}\left(a_{r}\right)+O\left(x^{-K}\right)\right\}+O\left(e^{-K_{2} x}\right),
$$

(iv) $-\pi<\alpha_{r} \leqq-\pi / 2$,

$$
\begin{aligned}
& X_{1, r}^{-l} H_{r}\left(X_{1, r}\right)\left\{C_{14}^{\left(\psi_{1}\right)}\left(a_{r}\right)+O\left(x^{-K}\right)\right\} \\
& \quad+X_{0, r}^{-l} H_{r}\left(X_{0, r}\right)\left\{C_{14}^{\left(\psi_{2}\right)}\left(a_{r}\right)+O\left(x^{-K}\right)\right\}+O\left(e^{-K_{2} x}\right) .
\end{aligned}
$$

For $x$ sufficiently large we have $|U|<\rho_{N}$, so that in case (i) the $\operatorname{arc} \Lambda_{4}(0)$ may be replaced by $\Gamma_{6}\left(U_{0}\right)$ together with the straight lines $L_{1}, L_{2}$ given by $|U| \leqq|s| \leqq \rho_{N}$, arg $s= \pm\left(\pi / 2+\xi_{N}\right)$, and the arc $\Lambda_{6}$ of the circle $|s|=|U|$ on which $\pi / 2+\xi_{N} \leqq \arg s \leqq \arg U_{0}+C_{15}\left(a_{r}\right)$. Then the result for $I_{l}^{(1)}\left(a_{r}, x\right)$ follows from Lemma 4 (iii) and (5.16), the integrals along $L_{1}, L_{2}$, and $\Lambda_{6}$ contributing only to the error term. Case (ii) is proved similarly, replacing $\Lambda_{6}$ by the arc $\Lambda_{6}^{\prime}$ on which

$$
\arg U_{0}-C_{15}\left(a_{r}\right) \leqq \arg s \leqq-\pi / 2-\xi_{N} .
$$

For case (iii), we first note that the contour $\Lambda_{4}(0)$ may be replaced by two curves $\Lambda_{4}^{(1)}(0), \Lambda_{4}^{(2)}(0)$, on which $\psi=\psi_{1}, \psi=\psi_{2}$ respectively. These curves join the origin to the end-points of $\Lambda_{4}(0)$, with $|s| \leqq \rho_{N}$ on each and $\alpha_{r}-\pi \leqq$ arg $s$ $\leqq \pi / 2+\xi_{N}$ on $\Lambda_{4}^{(1)}(0),-\pi / 2-\xi_{N} \leqq \arg s \leqq \alpha_{r}-\pi$ on $\Lambda_{4}^{(2)}(0)$. Then, if the straight lines $L_{3}, L_{4}$ are given by $|s| \geqq \rho_{N}$, arg $s= \pm\left(\pi / 2+\xi_{N}\right)$, the contour formed by $\Lambda_{4}^{(1)}(0)$ and $L_{3}$ may be replaced by $\Gamma_{4}\left(U_{0}\right)$, and that formed by $\Lambda_{4}^{(2)}(0)$ and $L_{4}$ by $\Gamma_{5}\left(U_{-1}\right)$ (the verification of these statements follows the lines of $\S 5$ of [10]). The result then follows by Lemma 4 (i) and (ii) and (5.15), the integrals along $L_{3}, L_{4}$ contributing the error term $O\left(e^{-K_{2} x}\right)$. Case (iv) is proved similarly, using the contours $\Gamma_{4}\left(U_{1}\right)$ and $\Gamma_{5}\left(U_{0}\right)$.

LemMa 6.

$$
I_{l}^{(2)}\left(a_{r}, x\right)=O\left(x^{-P-l-1}\right) .
$$

The integral may be related to a similar integral round the contour $\Lambda_{\mathbf{4}}^{\prime}(0)$ given by $|s|=\rho_{N} x,|\arg s| \leqq \pi / 2+\xi_{N}$, and the result follows from the order relation (5.14) for $\Omega(s)$.

Lemma 7. (i) If $\tau(s)$ has a simple zero at $s=a_{r}$, then

$$
\begin{aligned}
x^{\beta_{r}+l} I_{l}\left(a_{r}, x\right) & =C_{6}\left(a_{r}\right)\left\{\Gamma\left(1-\beta_{r}-l\right)\right\}^{-1}+O\left(x^{-K}\right) & \left(\beta_{r} \neq 1,2, \cdots\right), \\
& =(-1)^{M_{r}+l} \Gamma\left(M_{r}+l\right) C_{8}\left(a_{r}\right)+O\left(x^{-K}\right) & \left(\beta_{r}=M_{r}\right) .
\end{aligned}
$$

(ii) If $\tau(s)$ has a double zero at $s=a_{r}$ and $\vartheta\left(a_{r}\right)=0$, then 


$$
\begin{array}{r}
x^{\gamma_{r}-1} I_{0}\left(a_{r}, x\right)=C_{10}\left(a_{r}\right)\left\{\Gamma\left(2-\gamma_{r}\right)\right\}^{-1}+O\left(x^{-K}\right)+C_{11}\left(a_{r}\right) x^{\gamma_{r}-1} \\
\quad\left(\gamma_{r} \neq 1,2, \cdots\right), \\
=(-1)^{M_{r}-1} \Gamma\left(M_{r}-1\right) C_{12}\left(a_{r}\right)+O\left(x^{-K}\right)+C_{11}\left(a_{r}\right) x^{M_{r}-1} \\
\quad\left(\gamma_{r}=M_{r}>1\right), \\
=\log x\left\{-C_{12}\left(a_{r}\right)+O\left(x^{-K}\right)\right\}+C_{12}\left(a_{r}\right) \log \left(\begin{array}{c}
\left(-C_{5}\right)+C_{6}^{\prime \prime}\left(a_{r}\right) \\
\left(\gamma_{r}=1\right) .
\end{array}\right.
\end{array}
$$

(iii) With the conditions of case (ii), for $l=1,2, \cdots, n$

$$
\begin{aligned}
x^{\gamma_{r}+l-1} I_{l}\left(a_{r}, x\right) & =C_{10}\left(a_{r}\right)\left\{\Gamma\left(2-\gamma_{r}-l\right)\right\}^{-1}+O\left(x^{-K}\right) \quad\left(\gamma_{r} \neq 1,2, \cdots\right), \\
& =(-1)^{M_{r}+l-1} \Gamma\left(M_{r}+l-1\right) C_{12}\left(a_{r}\right)+O\left(x^{-K}\right) \quad\left(\gamma_{r}=M_{r}\right) .
\end{aligned}
$$

(iv) If $\tau(s)$ has a double zero at $s=a_{r}$ and $\vartheta\left(a_{r}\right) \neq 0$, then

$$
\begin{aligned}
I_{l}\left(a_{r}, x\right)= & \left(X_{r}^{\prime}\right)^{-l} H_{r}\left(X_{r}^{\prime}\right)\left\{C_{14}^{\left(\psi_{1}\right)}\left(a_{r}\right)+O\left(x^{-K}\right)\right\} \\
& +\left(X_{r}^{\prime \prime}\right)^{-l} H_{r}\left(X_{r}^{\prime \prime}\right)\left\{C_{14}^{\left(\psi_{2}\right)}\left(a_{r}\right)+O\left(x^{-K}\right)\right\} .
\end{aligned}
$$

Here $C_{6}{ }^{\prime}\left(a_{r}\right)$ is equal to $C_{6}^{\prime}\left(a_{r}\right)$ in (4.10) with $\tau^{\prime}\left(a_{r}\right), \beta_{r}$ replaced by $\tau^{\prime \prime}\left(a_{r}\right) / 2, \gamma_{r}$ respectively, and the other coefficients are as previously defined. The contour $\Lambda_{4}(0)$, together with the straight lines $L_{3}, L_{4}$ used in the proof of Lemma 5 , forms the Gamma function contour $\Lambda_{7}$. Integrals round $\Lambda_{7}$ may be related to similar integrals round $\Lambda_{7}^{\prime}$, which is the contour consisting of the arc $\Lambda_{4}^{\prime}(0)$, used in the proof of Lemma 6 , together with those portions of $L_{3}, L_{4}$ on which $|s| \geqq \rho_{N} x$. Then results (i)-(iii) are obtained by substituting for $Y_{02}(s)$ the expressions given in (4.2) or (4.7) for part (i) and in (5.1) or (5.2) for parts (ii) and (iii). From (5.2) we obtain two forms for $l=0$, since if $\gamma_{r}=1$ the integral

$$
\frac{1}{2 \pi i} \int_{\Lambda_{7}} s^{-1} \log s e^{s x} d s
$$

occurs, from which the term in $\log x$ arises. In case (iv), $Y_{02}(s)$ is given by (5.13), so that

$$
I_{l}\left(a_{r}, x\right)=I_{l}^{(1)}\left(a_{r}, x\right)+I_{l}^{(2)}\left(a_{r}, x\right)+O\left(e^{-K_{2} x}\right) .
$$

By the definition of $X_{r}, X_{r}^{\prime \prime}$ in $\S 2$ we have

$$
\begin{array}{llrl}
X_{r}^{\prime}=X_{0, r}, & X_{r}^{\prime \prime}=X_{-1, r} & \left(0 \leqq \alpha_{r} \leqq \pi\right), \\
X_{r}^{\prime}=X_{1, r}, & X_{r}^{\prime \prime}=X_{0, r} & \left(-\pi<\alpha_{r}<0\right) .
\end{array}
$$

For $\alpha_{r} \neq \pi$

$$
H_{r}\left(X_{j, r}\right)=H_{r}\left(X_{0, r}\right) O\left(x^{-K}\right) \quad(j= \pm 1),
$$


where the exponential factor $\exp \left(2 A_{r} X_{0, r}\right)$ in $H_{r}\left(X_{0, r}\right)$ has $R\left(A_{r} X_{0, r}\right)>0$, and for $\alpha_{r}=\pi$

$$
X_{r}^{-l} H_{r}\left(X_{r}\right)=O\left(x^{1 / 4-\gamma_{r} / 2-l / 2}\right) .
$$

Since the positive integer $P$ in the result of Lemma 6 may be arbitrarily large, we have

$$
I_{l}^{(2)}\left(a_{r}, x\right)=X_{0, r}^{-l} H_{r}\left(X_{0, r}\right) O\left(x^{-K}\right) .
$$

Thus result (iv) follows for all values of $\alpha_{r}$ from Lemma 5, and the proof of Lemma 7 is complete.

9. Proof of the theorems. By (7.1), (3.5), and (8.1), $y^{(\nu)}(x)$ is given for $\nu=0,1, \cdots, n-1$ by

$$
y^{(\nu)}(x)=\sum_{r=1}^{N} e^{a_{r} x} \sum_{l=0}^{\nu}\left(\begin{array}{l}
\nu \\
l
\end{array}\right) a_{r}^{\nu-l} I_{l}\left(a_{r}, x\right)+O\left\{e^{\left(a-K_{2}\right) x}\right\},
$$

and result (a) of Theorem 1 follows from Lemma 7 (i), with

$$
\begin{aligned}
C_{1}\left(a_{r}\right) & =C_{6}\left(a_{r}\right)\left\{\Gamma\left(1-\beta_{r}\right)\right\}^{-1} & \left(\beta_{r} \neq 1,2, \cdots\right), \\
& =(-1)^{M_{r}} \Gamma\left(M_{r}\right) C_{8}\left(a_{r}\right) & \left(\beta_{r}=M_{r}\right) .
\end{aligned}
$$

Here $C_{6}\left(a_{r}\right)$ is given by (4.3), (4.5), or (4.6) and $C_{8}\left(a_{r}\right)$ by (4.8).

Result (b) follows from Lemma 7 (ii), with

$$
\begin{array}{rlr}
C_{2}^{\prime}\left(a_{r}\right) & =C_{10}\left(a_{r}\right)\left\{\Gamma\left(2-\gamma_{r}\right)\right\}^{-1} \quad\left(\gamma_{r} \neq 1,2, \cdots\right), \\
& =(-1)^{M_{r}-1} \Gamma\left(M_{r}-1\right) C_{12}\left(a_{r}\right) \quad\left(\gamma_{r}=M_{r}>1\right), \\
C_{2}^{\prime \prime}\left(a_{r}\right)=C_{11}\left(a_{r}\right), \quad C_{2}\left(a_{r}\right) & =-C_{12}\left(a_{r}\right), \quad C_{3}\left(a_{r}\right)=C_{12}\left(a_{r}\right) \Gamma^{\prime}(1)+C_{13}\left(a_{r}\right) .
\end{array}
$$

Here $C_{10}\left(a_{r}\right), C_{11}\left(a_{r}\right), C_{12}\left(a_{r}\right), C_{13}\left(a_{r}\right)$ are equal to $C_{6}\left(a_{r}\right), C_{7}\left(a_{r}\right), C_{8}\left(a_{r}\right), C_{9}\left(a_{r}\right)$ respectively, where in each case $\tau^{\prime}\left(a_{r}\right), \beta_{r}$ are replaced by $\tau^{\prime \prime}\left(a_{r}\right) / 2, \gamma_{r}$ respectively, and $C_{7}\left(a_{r}\right)$ and $C_{9}\left(a_{r}\right)$ are given by (4.4) and (4.9) respectively.

The first part of result (c) follows from Lemma 7 (iv), with $C_{4}^{\prime}\left(a_{r}\right)$ $=C_{14}^{\left(\psi_{1}\right)}\left(a_{r}\right), C_{4}^{\prime \prime}\left(a_{r}\right)=C_{14}^{\left(\psi_{2}\right)}\left(a_{r}\right)$, where $C_{14}^{(\psi)}\left(a_{r}\right)$ is given by (5.5) if $\left|\alpha_{r}\right| \neq \pi / 2$ and by (5.6a), (5.6b) if $\alpha_{r}=\pi / 2,-\pi / 2$ respectively. Using (8.2) and (8.3), the second part is seen to be an immediate corollary of the first, with $C_{4}\left(a_{r}\right)$ $=C_{14}^{(\psi)}\left(a_{r}\right)$ where $\psi=\psi_{1}$ for $\alpha_{r} \geqq 0$ and $\psi=\psi_{2}$ for $\alpha_{r}<0$. This completes the proof of Theorem 1.

If $a_{r}=0$ for $r=p$, where $p$ takes any one of the values $1,2, \cdots, N$, the corresponding term in the summation with respect to $r$ in $(9.1)$ is $I_{v}(0, x)$. The results of Theorem 2 then follow, using Lemma 7 (iii) for case (b).

For Theorem 3 we note that, by Theorem 2 of [7], the additional conditions, that $y^{(n)}(x)$ be continuous and of bounded variation in $\left(0, b_{m}\right)$ and that the same be true of $v(x)$ in every finite interval $\left(0, x_{0}\right)$, imply that these conditions are satisfied by $y^{(n)}(x)$ in $\left(0, x_{0}+b_{m}\right)$. Thus the results (6.2), (6.4), and 
(6.5), and hence also (7.1), may be shown to hold for $\nu=n$, and the extension of Theorems 1 and 2 is immediate.

Since the conditions of these three theorems imply $\omega(v)<\omega_{n}(y)$, we conclude with a brief reference to the results which may be obtained if $\omega(v)$ $=\omega_{n}(y)$. The most interesting case is that in which the transform $V(s)$ has a singularity at a zero $s=a_{r}$ of $\tau(s)$, arising from the form of $v(x)$ for large positive $x$. Then Theorems 4, 5 of [2, pp. 194-196], and calculations similar to those of $\S \S 4,5$, and 8 of the present paper produce results of which the following is typical.

Suppose that $v(x)$ is $L^{2}\left(0, x_{0}\right)$ for all finite $x_{0}>0$ and that for $x \geqq K$ either

$$
v(x)=B x^{\beta} e^{a_{r} x}+g(x)
$$

or

$$
v(x)=B x^{\beta} e^{a_{r} x} \log x+g(x),
$$

where $B, \beta$ are independent of $x$ and the Laplace transform of $g(x)$ has abscissa of convergence less than $R\left(a_{r}\right)$. Then if $a_{r}$ is a simple zero of $\tau(s)$ the asymptotic expansion of $y^{(v)}(x)$ contains a term of the order of $v(x)$ at infinity if $R\left(\beta+\beta_{r}\right) \geqq 0$, together with a term of the order of $v(x) \log x$ if $\beta+\beta_{r}=0$. For a double zero at which $\vartheta\left(a_{r}\right)=0$, the expansion contains a term of the order of $x v(x)$ if $R\left(\beta+\gamma_{r}\right) \geqq 0$ and $R(\beta) \geqq-1$, together with a term of the order of $x v(x) \log x$ if $\beta+\gamma_{r}=0$ and $R(\beta) \geqq-1$ or if $\beta=-1 \geqq-R\left(\gamma_{r}\right)$, and also a term of the order of $x v(x)(\log x)^{2}$ if $\beta=-1=-\gamma_{r}$.

\section{REFERENCES}

1. K. L. Cooke, The asymptotic behavior of the solutions of linear and nonlinear differentialdifference equations, Trans. Amer. Math. Soc. vol. 75 (1953) pp. 80-105.

2. G. Doetsch, Theorie und Anwendung der Laplace-Transformation, New York, 1943.

3. G. Hoheisel, Lineare funktionale Differentialgleichungen, I. Mitteilung, Math. Zeit. vol. 14 (1922) pp. 35-98.

4. A. A. Mirolyubov, Solution of differential-difference equations with linear coefficients, (Doklady) Akad. Nauk SSSR (N.S.) vol. 85 (1952) pp. 1209-1210 (Russian).

5. E. C. Titchmarsh, Theory of functions, Oxford, 1932.

6. D. V. Widder, The Laplace transform, Princeton, 1946.

7. E. M. Wright, Linear difference-differential equations, Proc. Cambridge Philos. Soc. vol. 44 (1948) pp. 179-185.

8. 1 , The linear difference-differential equation with asymptotically constant coefficients, Amer. J. Math. vol. 70 (1948) pp. 221-238.

9. - The linear difference-differential equation with constant coefficients, Proceedings of the Royal Society of Edinburgh, vol. 62 (1949) pp. 387-393.

10. E. M. Wright and B. G. Yates, The asymptotic expansion of a certain integral, Quart. J. Math. Oxford (2) vol. 1 (1950) pp. 41-53.

Royal Holloway College, University of London, LONDON. ENGLAND 